\title{
Brief Analysis of the Visual Language of Digital Animation
}

\author{
Chunhua Zhao \\ School of Fashion Communication \& Fashion Communication Research Center \\ Beijing Institute of Fashion Technology, Beijing, China
}

\begin{abstract}
Digital animation is a new form of artistic expression of animation, which fully coincides with the cultural atmosphere and social context of pursuing visual perception in modern art. The visual language takes color, shape, space, motion as communication means to make appropriate expression in a specific context and carry out specific information transmission. Therefore, digital animation is not only an artistic product, but also a trend of visual communication exploration. It combines science and technology with art, providing more possibilities for artistic creation. This paper takes the visual language as the main line and attempts to explore the characteristics, performance and formation mechanism of digital animation.
\end{abstract}

\section{INTRODUCTION}

With the rapid development of information technology, digital animation has become the pillar product of animation industry. At the beginning of the 21 st century, animation is still dominated by comic magazines, single edition comics, TV animation films, cosplay, etc., and now the leading position of animation has been replaced by various digital animation products. In recent years, a number of high-quality domestic animation works have appeared, such as "Ne Zha: I am the Destiny" and "Jiang Ziya". Digital animation has attracted attention from all walks of life.

Digital animation is the use of digital media technology, which integrates text, sound, graphics and images, etc., transforms the abstract digit into the perceivable, watchable, manageable and interactive form of information, improves the production efficiency by copying and diversifies the visual expression by digital images and virtual reality technology. As a product of network and digital technology development, digital animation merges science and technology, humanities and art, and brings audience strong artistic impact and visual impression.

\section{Concepts And Explanation Of The IMAGE AND THE VISUAL LANGUAGE}

Visual language takes color, shape, space and movement as communication means to make appropriate expressions in a specific context and carries out specific information transmission. Digital technology ingeniously improves the expression level of visual language in a series of links such as modeling design and drawing, scene design and drawing, tracing line, coloring, and special effects making, and finally generates super-real virtual images, which profoundly improves the picture quality of animation.
The semiotician W.J.T. Mitchell once defined "image" as a concrete representation of the object in which the image appears [1]. Visual image is to create meaning through the interactive relationship between images. In this way, the ideographic function and structural function of images are very important for the generation of image meaning [2]. Each visual style, no matter it is color, shape or space, can be regarded as a kind of language statement. Through the arrangement, collocation and structure of harmonious, balance, change and unified color and shape, the meaning can be conveyed systematically.

It can be said that the picture is the signifying tool to organize many objects in the image, therefore, it is the "image with content". From the ideographic point of view, an image is an organized meaningful entity and a semantic unit. Vision is a way of communication between communicator and audience through visual language. Visual transmission production itself is a process of visual language reconstruction, which involves visual aesthetics, visual law, psychological influence and other aspects. It uses visual images to convey information, and utilizes graphics, text and color as the basic elements of visual language to perform artistic expression [3].

\section{Visual Characteristics of Digital ANIMATION}

Animation is the process of making image "alive", which can not only clearly show the process of an event, but also show a vivid picture.

Compared with traditional animation, digital animation shows many differences. With the help of digital technology, the scope and skills of expression in animation greatly expands. The color, line, composition and artistic conception of the picture becomes more diverse. The visual elements in the picture are arranged and combined with more aesthetic effect comparable to

zhaochunhua8989@sina.com 
that of the film. The combination and scientific collocation can effectively improve the visual appeal and bring the audience an immersive visual experience. Digital animation is generally manifested as the following characteristics:

\subsection{Aesthetics}

The colors, lines and forms of digital animation are freer and more diversified, which can be exquisite and exaggerated, and the picture is more ornamental. The application of computer digitization is based on the real images collected by real people, and it can make the real images more beautiful, more appealing and aesthetical, so as to continuously achieve artistic transcendence.

\subsection{Simulation}

The precise modeling ability of digital animation and rich expressive force can more realistically imitate the natural images of people and things visible, tangible, as if the real world "simulation" appears. And digital images can show the macroscopic world as well as the microscopic world, or from the universe to molecules.

\subsection{Virtualization}

Digital animation is a virtual existence, or a special form of reality. People, objects, scene digital, virtual reality high simulation images enhance the sense of integration, such as virtual natural phenomena, simulation of scientific phenomena, simulation of ancient civilization, virtual social life scene, fantasy world, underwater world. It is the full liberation of imagination with the support of information technology.

\section{Analysis of the Visual Language of Digital ANimation}

Digital animation is a new language of animation, which has its own special artistic style. In terms of image aesthetics, simulation and virtualization, visual language elements such as color, shape, space and movement have been fully applied, pushing the subjectivity, artistic aesthetics and artistic expression of animation to a new height.

The structural collocation of objects in animation pictures, the choice of colors, the coordination of the positions among foreground, background, subject and companion body, as well as the direction of lines, light and shade of film tones, the contrast between virtual and real or other composition factors can make a certain relationship between numerous objects and images, which can be integrated into an orderly indicating field [4]. The specific analysis is as follows:

\subsection{Color and Shape}

Color and shape are recognizable and symbolic. They are the basic elements in character modeling and the most important part in visual languages. Arnheim once said: It is not surprising that shapes have traditionally been compared to manly men and that colors have been compared to seductive women. The combination of shape and color is necessary to create the pictures [5].

The essential characteristics of color determine that it is often used to express personality, characteristics, identity, status and field, or are used to symbolize a nation's ideology, social system, religious belief and customs. These functions are somewhat similar to symbols, and their purpose is to communication visually. Color can reflect artistic style. Different color selection and hierarchy will be matched according to the priority of expression.

Shape or modeling, mainly refers to the use of artistic modeling means to create graphics. In addition to the structure of objective people and objects, modeling can also be extended to a larger scope, that is, consciously and actively driving the basic type, combination, reconstruction, arrangement in a limited space, and thus find more beauty. Through the refinement of reality, the artist artistically processes a large number of life materials based on stories and exaggerates them to create distinctive features, people and things that can promote the development of the plot.
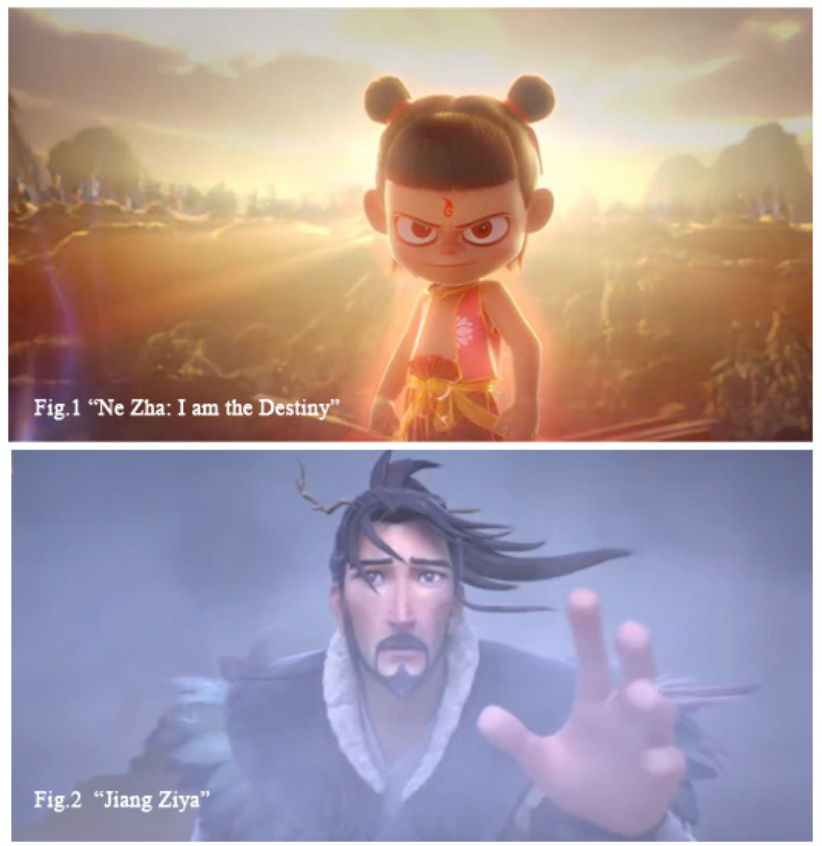

Taking "Ne Zha: I am the Destiny" (Fig. 1) and "Jiang Ziya" (Fig. 2) as examples, the former depicts the selftransformation and human sublimation of Ne Zha's thanks to the power of love. Therefore, although the protagonist Ne Zha is portrayed as a rebellious urchin, such as a loose walking posture with angry staring eyes, gold is used frequently as background color to give the audience warm sense, which echoes the theme "the goodness of human nature"and "the sublimation of human nature". The gold color in the background and the rebellious form of Ne Zha set off each other, making Ne Zha's evil spirit with a hint of warm goodwill. "Jiang Ziya" is an animation that reflects the complex psychological process of the individual's cognitive subversion of authority and rules after discovering the cruel truth. It is a film with a critical 
color. The fog, the blue-gray background and the hands of the protagonist stretching into the fog indicate the confusion and difficulty of the protagonist Jiang Ziya, and also imply the tragic ending.

Different from traditional animation, both of the two films use digital technology, which makes the color saturation higher, the lines more delicate, the picture more ornamental. The application of computer digitization is based on the real images collected, and the real images are processed more realistically through technical means. The simulation effects is almost achieved, even with threedimensional effects. The appeal of characters and scenes is stronger.

\subsection{Space}

The image is actually a concept of space, which expresses the meaning through the relationship between the images in the picture frame and the organization and composition of the images. Animation can not only reproduce the real space, but also reconstruct the new form of space. Through the selection, highlighting and recombination of visual information, the image forms a creative spatial form. It makes use of people's psychological mechanism to expand the space and bring the audience to a special world. Thus, the basic meaning of the image language is generated. Although the image is not the reality itself, it eliminates the estrangement between the picture and the reality to the greatest extent, allowing the audience to be on the scene.

In the animation, the spatial concept is used everywhere. It is interactive with the characters and plays a role in shaping the objective space and psychological space of the scene. Scene space is the expansion and extension of character. The introduction of the scene space strengthens the internal connection between the character form and the frame structure, and forms the sense of reality of the character space and the richness of the space expression. Moreover, the scene space indicates the spatial characteristics of characters' existence and activities. The specific and direct environmental performance confirms the existence state of the characters in the specific spatial relationship, and the specific spatial atmosphere plays an important role in reinforcing the character characteristics and emotional expression.

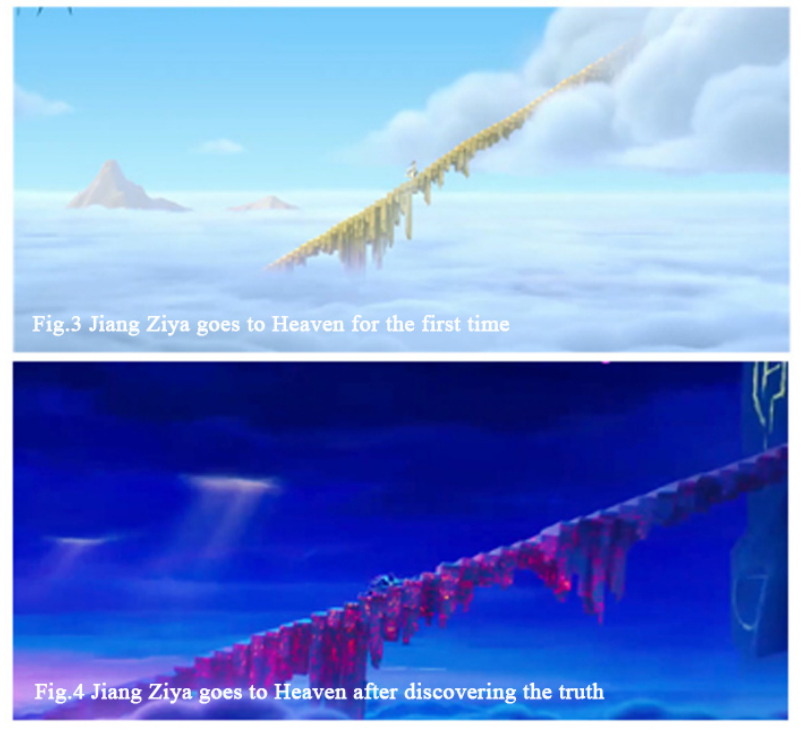

Take "Jiang Ziya" for example, the "Heaven Ladder" is not only the physical distance between Heaven and the Human, but also the reflection of the protagonist Jiang Ziya's mental journey. When the hero goes to Heaven for the first time (Fig. 3) and after discovering the truth (Fig. 4), the celestial bodies are still there, while "the senses are different". The whole scene from color, character temperament to the structure of the ladder, has changed, which properly reflects the changes of the characters' inner world.

The precise modeling ability of digital technology, superior scene layout, special effects synthesis ability, produce this magnificent scene from imagination to reality Digital animation creates a "fantasy" that some traditional art can't show, and achieves the effect of "virtual reality". Sometimes, "virtual reality" created by computers sometimes appears more realistic, more infectious, more sensory impact, with surreal, dreamlike effect.

\subsection{Motion}

Animation is an art based on motion and speed. As a basic means of animation, motion change is an important symbol of the form of animation ontology, which realizes motion modeling through symbol change, and the form of motion is presented in the form of displacement and deformation in animation. The word "animation" originally means "to give life", i.e., animation gives life to the picture through technical means and makes the lifeless picture come alive.

Motion is undoubtedly the essential element and aesthetic basis of animation, and the visibility and movement of the picture are the basic conditions for animation. This is not only reflected in the time latitude, but also reflected in the speed scene conversion, character modeling, scene scheduling, color and light conversion. Different from the shooting techniques of ordinary film and television works, animation is the motion form of things created by human beings with the help of technical means. Animation syntheses kinesthetic movement of the pictures by continuous rapid movement (interval duration between approximately $1 / 20-1 / 10$ second) of the 
"relevant" pictures [6]. Animation creates dynamic vision by shooting a series of individual images on a continuous, multi-lattice film. In digital frame animation, acceleration and deceleration, delay and pause, the change of character position, as well as changes in shape affect the audience's kinesthetic cognition. In the flow of time, animation exhibits aesthetics of changes in a dynamic way, or expresses the flow thoughts and consciousness. Thus, animation has its unique artistic characteristics and visual language rules. It can freely show the changes of people, animals, objects, scenes, with exaggeration and fantasy which can hardly fulfil in reality.

Animation is a language system that constantly creates artistic conception of movement. Through digital virtual technology, animation creators obtain virtual space environment, motion capture tracks and changing light and shadow effect. By the use of non-lens technology, we get a plenty of new experience to establish the moving lines [7]. Virtual technology, as a computer simulation system, provides audience with virtual simulation visual perception by combining simulation technology, computer technology and microelectronics technology. As the most intuitive ideographic interface of animation, dynamic visual screen plays a major role in flow of the story under the mimicry environment.

For example, in the virtual mirror "the Picture of Mountains and Rivers in the Kingdom " (Fig. 5, 6 ,7) in "Ne Zha: I am the Destiny", Taiyi Immortal takes Ne Zha to travel through the sky and sense the wonder of the fairyland. This scene, from the lotus pond to the cascading waterfall, from the spreading vines to the swift boat wandering in the waterfall flowing in the sky, is produced by the staff with the use of exaggerated movement modeling, action trajectory, speed changes, scene conversion, special effects and other technical means, to create a stunning visual spectacle.
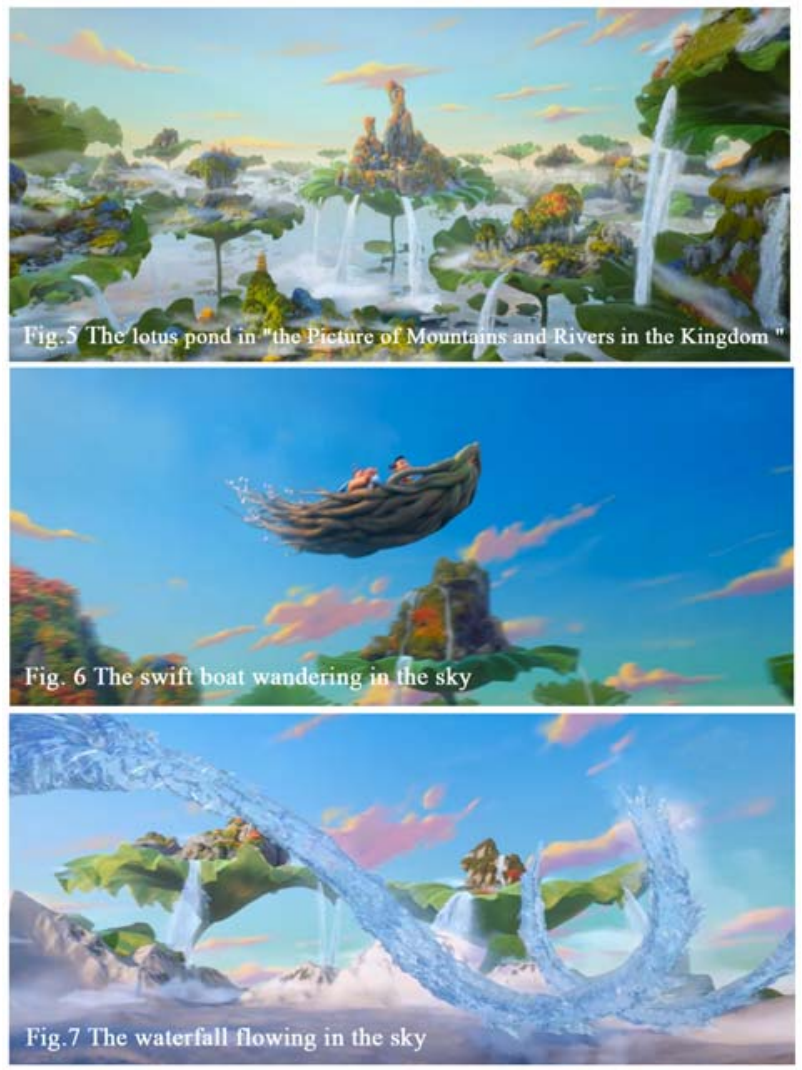

Under the digital condition, we obtain the desirable scene phenomenon and the motion speed and trajectory of the subject in accordance with the natural motion rule through digital modeling and data control, thus create a mimicry environment with high degree of authenticity. In terms of the structure of characters' movement trajectories, digital technology adopted skeletal animation technology to conduct behavior control for each character in the virtual animation

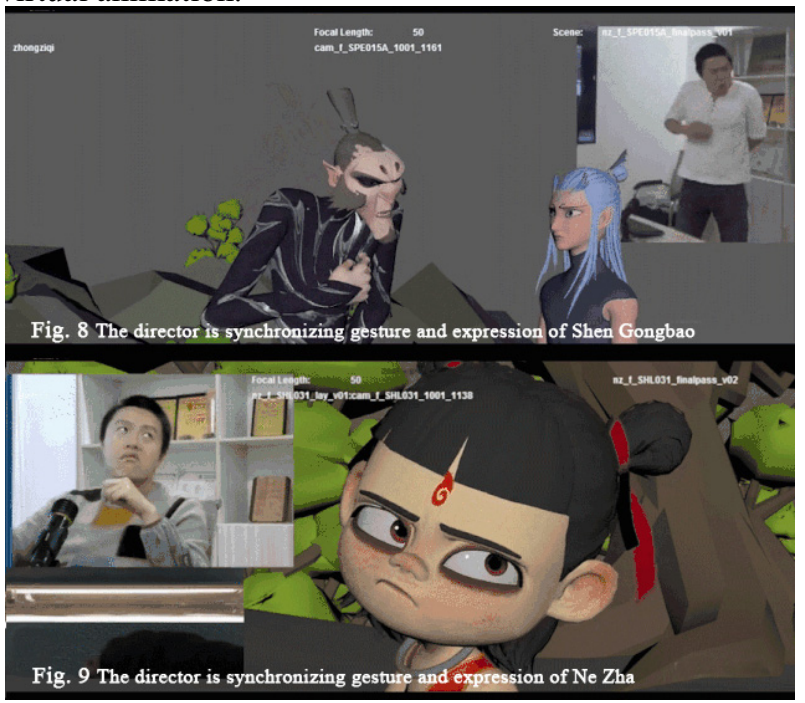

For example, the above pictures shows the director synchronizing the gestures and the expressions of Shen Gongbao (Fig. 8) and Ne Zha (Fig. 9) in "Ne Zha: I am the Destiny" with the help of 3D digital image technology. Motion capture technology is used in the virtual animation design to improve the convenience and lifelike effect of the animation motion [8]. 
Due to the application of digital technology, the narrative style and artistic language of animation have undergone great changes. The rapid development of technology has broken the physical limits of creation. With the strong technical support, the animation modeling and display ability has been greatly expanded. It not only inherits the advantages of spatial layout in traditional animation, but also absorbs the elements of movement in visual art, adds new aesthetic dimension of time flow in expression, and highly enhances the authenticity of the product, the integrity of the process and the impact of special effects.

\section{CONCLUSION}

Digital animation is a new form of animation, which fully coincides with the cultural atmosphere and social context of pursuing visual perception in modern art. Therefore, digital animation is not only an artistic product, but also a trend of visual communication exploration. It integrates science and technology with art, providing more possibilities for artistic creation. Especially, its visual design language, practice and research scope have been further promoted and extended.

\section{ACKNOWLEDGMENT}

This paper was the phased research results of the Social Science Project of Beijing Education Commission (No.: SM201810012003) and the Special Fund Project for the Construction of High-level Teachers Team of Beijing Institute of Fashion Technology (No.: BIFTTD201803).

\section{REFERENCES}

1. W.J.T. Mitchell, Image Theory, Beijing: Peking University Press, 2006, pp. 4.

2. Danian Zhong and Jianjun Lei, Documentary: The Meaning System of Images, Beijing: Beijing Normal University Press, 2012, pp. 207.

3. Ming Gao, Principles of Advertising and Visual Communication, Shanghai: Wenhui Press, 2008, pp. 8.

4. Danian Zhong and Jianjun Lei, Documentary: The Meaning System of Images, Beijing: Beijing Normal University Press, 2012, pp. 17-23.

5. Jichun Huang and Tao Zhao, "The Application of Color in the Modeling of Animated Characters," Modern Marketing, vol.7, pp.253, July 2012.

6. Baojun Guo, Fengmei Yang and $\mathrm{Na}$ Zhao, "The Modification of Animation Principle _ Based on the Animation Principle of the Joint Action of Visual Retention and Animated Phenomena", Film and Television Production, pp. 11, September 2009.

7. Xiangyu Wang, "Image Motion Modeling and Digital Visual Communication of Chinese Contemporary Films", Contemporary Films, vol.6, pp. $151,2007$.
8. Wenyue Jin, "Research on Character Modeling and Behavior Control Based on Virtual Animation Design", Modern Electronic Technology, Vol. 43, pp. 111-112, September 2020.

9. Chunhua Zhao, Fashion Communication, Beijing: China Textile Press, 2018, pp. 117-123. 